\title{
Through the LAMS towards the future: current uses and outcomes of lumen-apposing metal stents
}

\author{
Alessandro Mussetto ${ }^{a}$, Alessandro Fugazzab ${ }^{b}$ Lorenzo Fuccioc, Omero Triossi ${ }^{a}$, Alessandro Repicib, \\ Andrea Anderloni ${ }^{\mathrm{b}}$
}

S. Maria delle Croci Hospital, Ravenna; Humanitas Research Hospital, Rozzano, Milan; S.Orsola-Malpighi Hospital, University of Bologna, Bologna, Italy

\begin{abstract}
The lumen-apposing metal stent (LAMS) is one of the revolutionary devices recently developed for gastrointestinal endoscopy. This device has a saddle-shaped design and large lumen. It was originally designed for drainage of transmural pancreatic fluid collection and in the last few years it has been used extensively for that indication. More recently, other in- and off-label indications have been proposed. Several types of LAMS are available, with or without an electrocauteryenhanced delivery system. In the current review we discuss the state of the art with regard to LAMS and their indications, usage, and outcomes.
\end{abstract}

Keywords Lumen-apposing metal stents, pancreatic fluid collections, endoscopic ultrasoundguided biliary drainage, endoscopic ultrasound-guided gastroenterostomy

Ann Gastroenterol 2018; 31 (5): 1-6

\section{Introduction}

The lumen-apposing metal stent (LAMS) is a recently developed device. It has a "barbell" shape with flanged ends that give it a theoretically very low risk of migration. This stent was originally created for pancreatic fluid collection (PFC) drainage, because of its larger inner lumen diameter than either plastic or traditional self-expanding metal stents. In addition, it allows for an endoscope to pass into collections to perform direct necrosectomy. Over time, many indications have been proposed.

There are several different LAMS systems available at this time, with different lengths and diameters (Aixstent, Axios, Hot Axios, Nagi, Spaxus, Hanaro-stent and Microtech-stent for pseudocysts) (Fig. 1). The diameter and length measurements vary between $6 \times 8 \mathrm{~mm}$ and $16 \times 30 \mathrm{~mm}$. The first described and most widely used and studied is the Axios system, "HOT", in

${ }^{\mathrm{a}}$ Gastroenterology Unit, S. Maria delle Croci Hospital, Ravenna (Alessandro Mussetto, Omero Triossi); bigestive Endoscopy Unit, Division of Gastroenterology, Humanitas Research Hospital, Rozzano, Milan (Alessandro Fugazza, Alessandro Repici, Andrea Anderloni); 'Department of Clinical Medicine, S. Orsola-Malpighi Hospital, University of Bologna, Bologna (Lorenzo Fuccio), Italy

Conflict of Interest: None

Correspondance to: Alessandro Mussetto, Ospedale Santa Maria delle Croci, Viale Randi 5, 48100 Ravenna, Italy,

e-mail: alessandromussetto@gmail.com

Received 14 March 2018; accepted 30 April 2018;

published online 26 June 2018

DOI: https://doi.org/10.20524/aog.2018.0287 the novel electrocautery-tipped delivery version (EC-LAMS).

This manuscript will review the current state of the art with regards to LAMSs and their indications.

\section{Technical aspects of EC-LAMS}

The use of a LAMS designed for transluminal drainage was first reported by Binmoeller and Shah in 2011 [1]. The AXIOS stent (Boston Scientific, Marlborough, MA, USA, Fig. 1) was designed to provide anchorage across non-adherent luminal structures. The handle of the AXIOS delivery system is Luerlocked onto the echoendoscope channel, analogous to a standard fine needle. The mechanism of release of the LAMS with an electrocautery-enhanced delivery system allows puncture and release of the stent in a single-step procedure, thus decreasing the number of accessories to be exchanged and consequently potentially reducing the frequency of complications. In fact, cautery enables transmural advancement of the stent delivery catheter into the target lumen without prior needle or guidewire insertion or preliminary dilation. In addition, while endoscopic-guided drainage has traditionally utilized fluoroscopy to optimize visualization, the EC-LAMS system has the potential to be deployed safely and effectively without fluoroscopic guidance, using ultrasound alone. Immediately after entry into the target lumen, the apposing stent is easily deployed under endoscopic ultrasound (EUS) and endoscopic guidance. The first clinical use of this device was described by Teoh, Binmoeller and Lau [2]. 


\section{PFCs}

PFCs are a frequent complication of acute pancreatitis and are classified into pancreatic pseudocysts (PP) and walled-off pancreatic necrosis (WON). Symptomatic PFC requires drainage options that include endoscopic approaches. Endoscopic drainage has emerged as the first-line therapy in the management of PP and WON and has significant advantages compared with surgical and percutaneous drainage [3]. EUS-guided drainage by use of plastic stents or self-expanding metal stents (SEMS) has been well established, but some concerns have been raised about the small diameter of the first and the risk of migration of the second.

LAMS have been shown to be efficacious for endoscopic transmural drainage of PP and WON (Fig. 2,3). Reports in

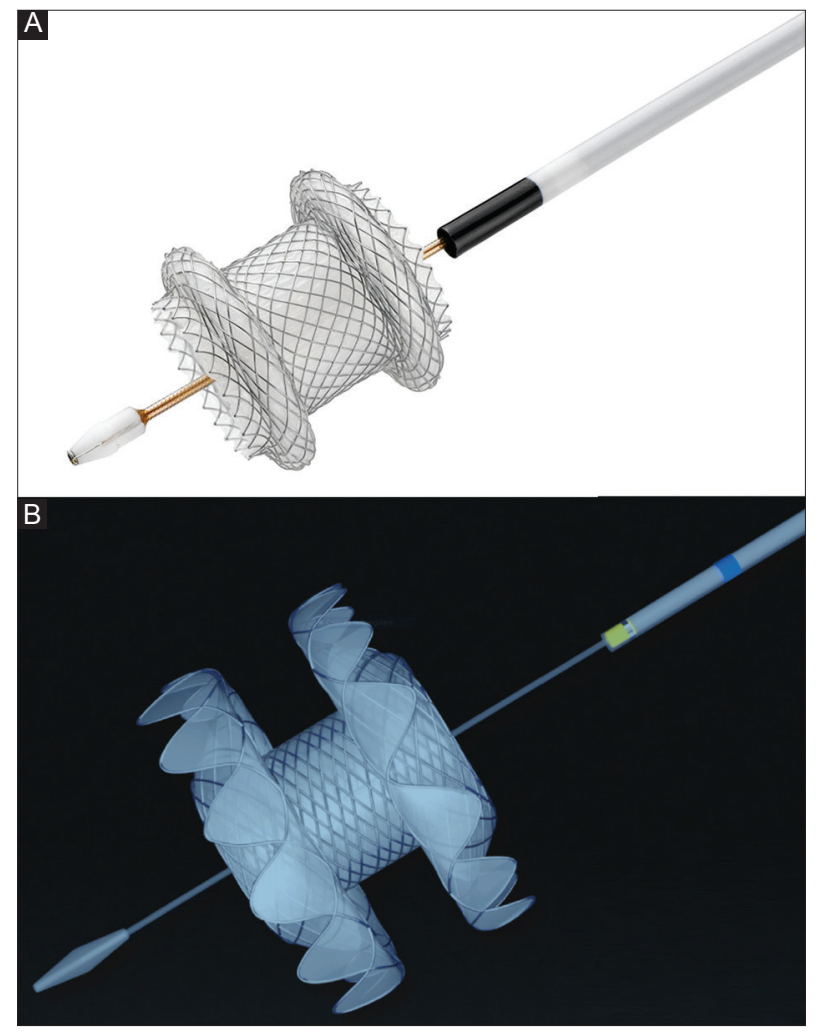

Figure 1 Examples of lumen-apposing metal stents. (A) HOT AXIOS stent (Boston Scientific), (B) Nagi stent (Taewoong Medical Co. Ltd.). Adapted from Boston Scientific and Taewoong Medical Co. Ltd. the literature concerning LAMS and PFC are continually increasing (Table 1) [4-10]. A recent meta-analysis [11], including technical and clinical success rate, and percentage of adverse events, included 14 studies from 2012 to 2016 and 812 patients (608 WON, $204 \mathrm{PP}$ ). For drainage of WON, the pooled technical success was $98.9 \%$ and clinical success was $90 \%$. For drainage of PP, the pooled technical success was $97 \%$ and clinical success was $98 \%$. More recent studies confirmed the good efficacy of the LAMS in the management of PFCs, even in the "electrocautery-enhanced fluoreless" version. Yoo et al identified 25 patients with PFC (3 PP, $22 \mathrm{WON}$ ) in whom EUS-guided transmural drainage was performed using ECLAMS. Technical success with placement of the EC-LAMS was achieved in all 25 patients. There were no procedure-related complications. PFC resolved in 24 patients (96\%) [10].

Some studies compared the efficacy and the safety of LAMS and other stents in the treatment of PFC. A multicenter retrospective study by Siddiqui et al [9] showed that endoscopic drainage and debridement of WON using LAMS was superior to double-pigtail plastic stents (DPPS) in terms of overall treatment efficacy. However, no significant differences were observed in WON resolution when comparing LAMS with fully covered SEMS (even though the mean number of procedures required for WON resolution was significantly lower with LAMS than with fully covered SEMS). A single-center retrospective collection of data by Law et al [12] compared fully covered SEMS and LAMSs in the drainage of WON and revealed that, while the two stents were comparable in terms of clinical efficacy and safety of EUS-guided drainage and debridement, the LAMSs were associated with early revision necessitated by either migration, dislodgment during necrosectomy, or ineffective drainage. A single-center retrospective comparison between LAMS and DPPS was performed by Lang, including a total of 103 patients (84 DPPS, 19 LAMS) [13]. This analysis showed that DPPS and LAMS were equally effective treatments for PP and WON, but LAMS were associated with higher rates of adverse events (53\% vs. $11 \%$ ), specifically bleeding.

Recently, more concerns have been raised about complications of LAMS. An interim analysis of an ongoing trial [14] showed a high rate of adverse events in patients with WON treated using LAMS vs. WON treated with plastic stents ( 6 patients vs. 0 patients, $50 \%$ vs $0 \%$ ). None of these adverse events were encountered intraprocedurally; they manifested late or were evident only at the time of clinical follow up.

Table 1 Comparison of main studies using lumen apposing metal stents for pancreatic fluid collections

\begin{tabular}{|c|c|c|c|c|c|c|}
\hline Author [ref.] & $\mathrm{N}^{\circ}$ of patients & PP & WON & Technical success rate (\%) & Clinical success rate $(\%)$ & $\begin{array}{c}\text { Adverse event rate } \\
(\%)\end{array}$ \\
\hline Rinninella [4] & 93 & 18 & 75 & WON 98.7 PP 100 & WON 90.7 PP 100 & 5 \\
\hline Shah [5] & 33 & 22 & 11 & WON 81.8 PP 95.5 & WON 63.6 PP 95.5 & 15.2 \\
\hline Siddiqui [6] & 82 & 14 & 68 & WON 100 PP 100 & WON 88.2 PP 85.7 & 9.8 \\
\hline Walter [7] & 61 & 15 & 46 & WON 97.8 PP 92.9 & WON 81.4 PP 100 & 9 \\
\hline Sharaiha $[8]$ & 124 & 0 & 124 & WON 100 & WON 86.3 & 11.3 \\
\hline Siddiqui [9] & 86 & 0 & 86 & WON 97.7 & WON 89.5 & 12.8 \\
\hline Yoo [10] & 25 & 3 & 22 & WON 100 PP 100 & WON 95.4 PP 100 & 8 \\
\hline
\end{tabular}

WON, walled-off pancreatic necrosis; PP, pancreatic pseudocsts 


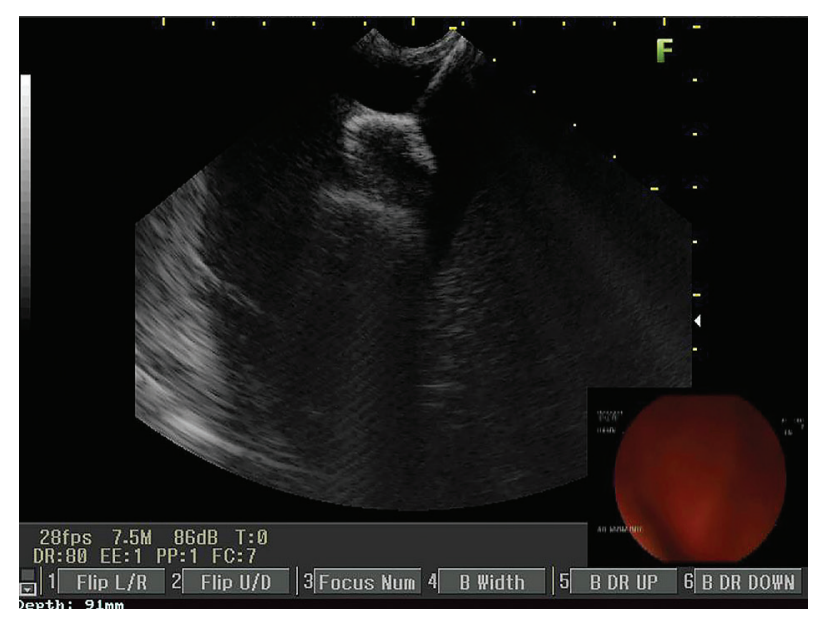

Figure 2 Endoscopic ultrasound image $(7.5 \mathrm{MHz})$ of a large pancreatic fluid collection with solid and liquid contents. HOT AXIOS stent (Boston Scientific Corp.) on its delivery catheter before deployment

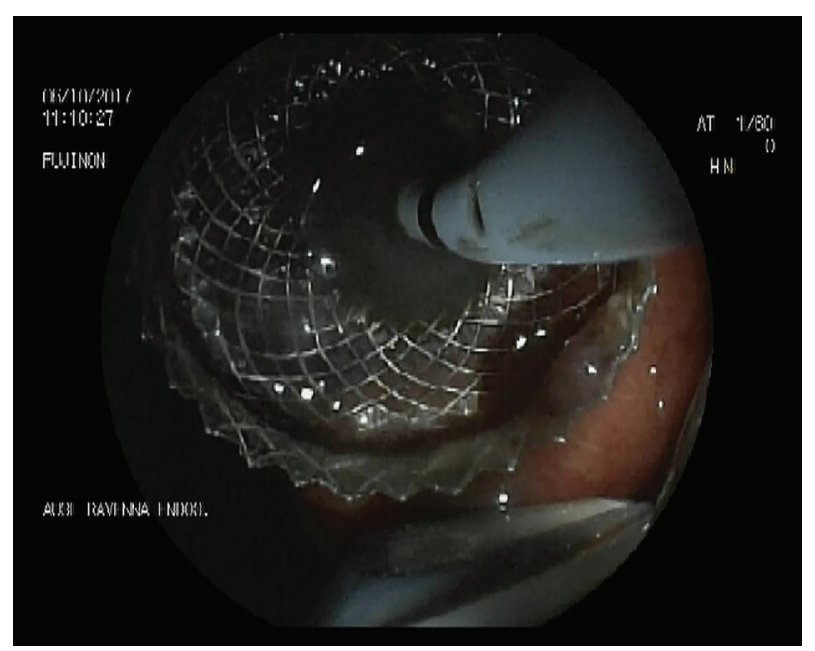

Figure 3 Lumen stent immediately after deployment

The adverse events were bleeding (3 patients), buried LAMS syndrome (2 patients) and biliary stricture secondary to mechanical compression of the stent (1 patient). In particular, delayed bleeding represents the most worrisome adverse event; in fact LAMS, with the resolution of collection, causes a friction against regional vasculature surrounding the necrotic cavity and this can leading to bleeding. The authors suggest performing an early computed tomography scan (no more than 3 weeks post LAMS placement) to evaluate the response, potentially followed by stent removal to prevent bleeding and buried syndrome [14].

In conclusion, the LAMS is an effective endoscopic device and has the potential to significantly simplify and streamline EUS-guided management of PFCs; this could help its widespread adoption as an alternative to surgery. With particular regard to the safety of these procedures, it seems to be mandatory to perform prospective randomized studies assessing risk factors for procedural adverse events of EUS-guided treatment of PFCs, including the role of direct necrosectomy.

\section{EUS-guided biliary drainage}

After gaining initial experience with draining pancreatic fluid collections, the indication for EUS-guided drainage has expanded to the biliary system, including EUS-guided hepaticogastrostomy, EUS-guided choledochoduodenostomy and EUS-guided cholecystostomy in particular, in cases where endoscopic retrograde cholangiopancreatography (ERCP) is not feasible. LAMS have an increasing role in the setting of choledochoduodenostomy (CDS) and gallbladder drainage (GBD).

\section{CDS}

The increasing interest in LAMS for EUS-guided CDS was stimulated by the lack of a dedicated metal stent. One of the first cases described was by Itoi and Binmoeller [15]; the stent was placed correctly without any adverse events in a patient with unresectable pancreatic cancer and a previous failed ERCP. In a retrospective study in seven tertiary European centers that included 57 patients with unresectable distal bile duct obstruction after ERCP failure, Kunda et al [16] demonstrated technically successful placement of EUS-guided CDS with AXIOS or Hot-AXIOS LAMS in 56/57 patients (98.2\%). A range of stent sizes was used. Clinical success was achieved in $54 / 56$ patients ( $96.4 \%$; $94.7 \%$ of entire cohort). The adverse event rate was $7 \%$ and included two duodenal perforations, one bleeding, and one transient cholangitis. Five patients required re-intervention for a stent migration and sump syndrome in 4 patients. At the end of follow up, the stent was patent without any intervention in 49/54 patients (90.7\%).

A more recent prospective study by Tsuchiya [17] evaluated the long-term outcome of EUS-CDS using LAMSs. Nineteen patients with unresectable malignant diseases were treated in 5 tertiary referral centers after a failed ERCP. EUS-CDS was performed using a fully covered LAMS with a cautery-enhanced delivery system. All stents were successfully deployed without any adverse events. Jaundice improved finally in $95 \%$. In $95 \%$ of patients the stents remained in good anastomotic position without migration or dislocation during a median six-month follow-up period. During follow-up, 5 patients had secondary stent obstruction because of food residue $(n=2)$, kinking $(n=1)$, suspected tumor ingrowth $(\mathrm{n}=1)$, and spontaneous dislodgement $(\mathrm{n}=1)$. The overall adverse event rate was $36.8 \%$, mostly with mild severity.

An approach for the palliation of patients presenting with simultaneous duodenal and biliary obstruction, based on the single-session sequential deployment of the novel cautery-tipped LAMS and a duodenal stent in patients with unreachable papilla or failed ERCP was also described, with optimal results [18].

In conclusion, the novel dedicated LAMSs have high technical and clinical success rates for EUS-CDS. The adverse events and patency rates may represent a limitation and further comparison studies with transpapillary stents are needed. In addition, stent design improvements are necessary to produce a more suitable and dedicated LAMS [17]. 


\section{EUS-guided GBD}

EUS-guided GBD (Fig. 4,5) using LAMS is still in development but has been performed in specific situations with very encouraging results. The indication remains the need for decompression of the gallbladder in patients unfit for surgery, in the setting of acute cholecystitis and/or biliary obstruction due to neoplastic conditions.

In a retrospective review of 15 non-surgical patients in 3 tertiary care centers who underwent EUS-GBD using the AXIOS LAMS to decompress the gallbladder, Irani et al [19] had technical success in $93.3 \%$ of patients and achieved clinical success in 15/15 patients over a median follow up of 160 days. Indications for the procedures included 7 patients with calculous cholecystitis, 4 with acalculous cholecystitis, 2 with biliary obstruction, 1 with gallbladder hydrops, and 1 with symptomatic cholelithiasis. One patient had post-procedure fever for 3 days; no other adverse events were noted. No patients had evidence of post-procedure bile leakage or stent migration.

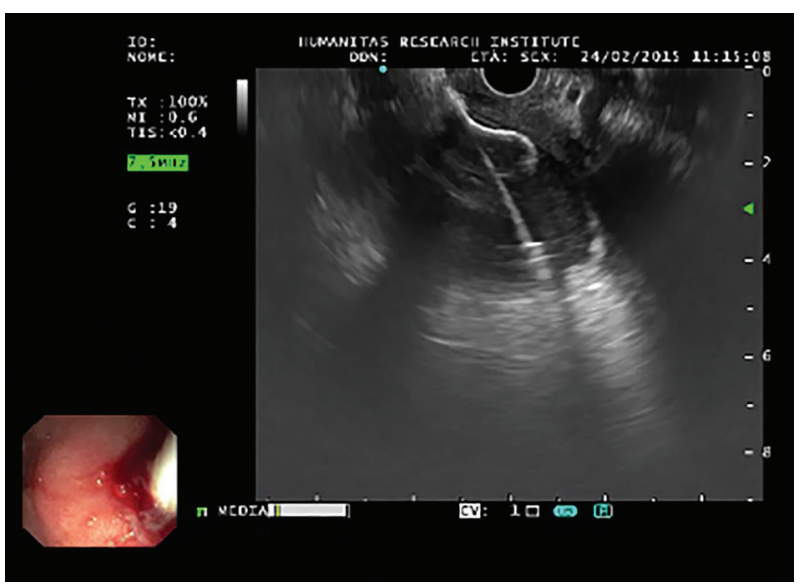

Figure 4 Endoscopic ultrasound image $(7.5 \mathrm{MHz})$ of drainage, performed from the duodenal bulb directly with the Hot Axios device (distal flange opened inside the gallbladder)

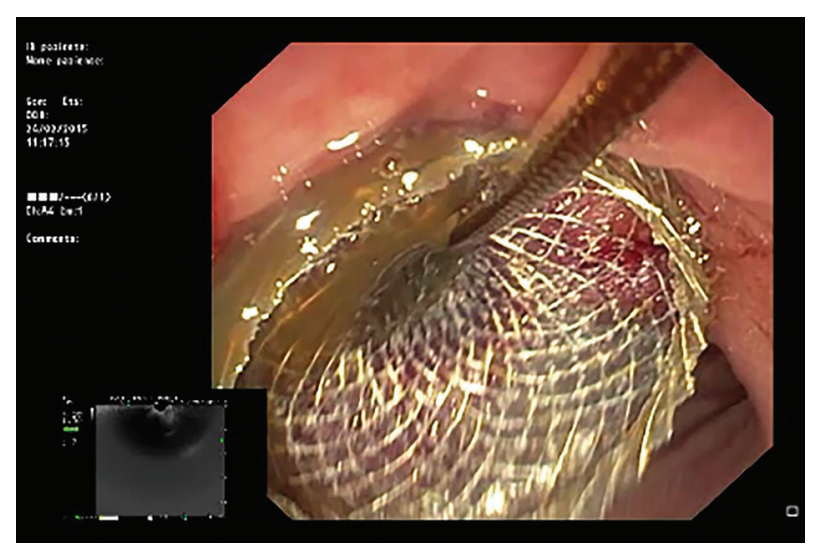

Figure 5 Endoscopic image showing pus draining through the stent from the gallbladder
The first multicenter prospective study of the use of a LAMS for EUS-GBD in high-risk surgical patients with acute cholecystitis was published by Walter et al [20]. Technical success was achieved in $27 / 30$ patients $(90.0 \%)$ and clinical success in $26 / 27$ patients $(96.3 \%)$. Interestingly, half of the patients did not undergo LAMS removal because of their poor functional status and/or because they declined a repeat procedure. These stents were left in place for an average time of 364 days, during which time no LAMS-related complications were observed. Regarding safety, the 30 -day mortality in the study was $17 \%$, comparable with the 30-day mortality or in-hospital death of $15.4 \%$ after percutaneous GBD (PTGBD). In addition, the 7\% stentrelated or procedure-related mortality observed in the study is comparable with that of PTGBD (around 4\%). However, the rate of non-fatal serious adverse events $(n=9,30 \%)$ is substantially higher than reported for PTGBD (15\%).

A recent multicenter study focused on GBD using LAMS. The authors retrospectively evaluated a large cohort of prospectively enrolled patients with acute cholecystitis and high surgical risk who underwent EUS-GBD using the newly available electrocautery-enhanced delivery system. The study showed technical and clinical success rates of $98.7 \%$ and $95.9 \%$, respectively, with procedure-related and short- and long-term adverse events occurring in $10.7 \%$ of the entire cohort. The authors reported serious stent- or procedure-related adverse events in $13 \%$ of patients [21].

A retrospective study of 118 patients that compared PTGBD (59 patients) and EUS-guided drainage with both AXIOS and HOT AXIOS devices (59 patients) found no differences in technical and clinical success between the PTGBD and EUSGBD arms, but the overall adverse events and severe adverse events were much more frequent in the PTGBD subgroup as compared with the EUS-GBD subgroup (74.6\% vs. $32.2 \%$, $\mathrm{P}<0.001$, and $74.6 \%$ vs. $23.7 \%, \mathrm{P}<0.001$, respectively) [22].

Similar conclusions (but with more advantages for EUSGBD) were drawn by Irani et al [23] in a retrospective study comparing EUS-GBD and PTGBD in 7 centers across USA, Europe and Asia. A total of 90 patients with acute cholecystitis underwent EUS-GBD $(n=45)$ or PTGBD $(n=45)$. No statistically significant differences were reported concerning the technical and clinical success. A nonsignificant trend toward fewer adverse events was appreciated in the EUS-GBD group. Patients who underwent EUS-GBD seemed to have shorter hospital stays, lower pain scores, and fewer repeated interventions.

Another field of research for LAMSs will surely be GBD for rescue treatment of malignant distal biliary obstruction after unsuccessful ERCP. In 2016 Imai et al [24] tested the use of SEMS in this setting with good results. The rates of technical success, functional success, adverse events, and stent dysfunction were $100 \%, 91.7 \%, 16.7 \%$, and $8.3 \%$, respectively.

Thus, EUS-guided transmural GBD is emerging as an attractive, elegant alternative to PTGBD for the management of acute cholecystitis in surgical high-risk patients. Moreover, "salvage" GBD with LAMS seems to be an attractive option in malignant obstructing conditions. Comparative controlled studies are required to confirm these results in terms of longterm outcomes and cost-effectiveness. 


\section{Other procedures}

\section{Gastroenterostomy (GE)}

EUS-guided GE has regained traction as a feasible, less invasive alternative to surgical bypass, fueled by the use of LAMS. In the direct EUS-GE technique, a 22-G needle is used for transgastric (TG) puncture into the lumen of the apposing jejunum and saline infusion is used to distend the bowel. A larger 19-G needle is then used to coil a wire within the distended jejunum, the gastroenterostomy tract is dilated over that wire, and the LAMS may be deployed across the tract. HOT-AXIOS is available to avoid the steps of TG puncture and wire placement.

Tyberg et al examined the utility of LAMS for EUS-guided gastrojejunostomy (EUS-GJ) in patients with benign and malignant gastric outlet obstruction (GOO). In an international, prospective trial of 26 patients with GOO, the authors demonstrated technical success using LAMS in 24 patients (92.3\%). The authors used EUS to identify a loop of small bowel beyond the level of obstruction and access it via EUS fine-needle aspiration and guidewire placement, allowing transluminal LAMS placement. Clinical success, in which patients were able to tolerate an oral diet, was achieved in 22 patients (84.6\%) [25].

In recent times, a direct comparison between EUS-GJ using LAMS and laparoscopic gastrojejunostomy (Lap-GJ) was performed in a multicenter study and 54 patients were enrolled [26]. Technical success was achieved in 29 (100\%) patients in the Lap-GJ group and $22(88 \%)$ in the EUS-GJ group $(\mathrm{P}=0.11)$. Clinical success was achieved in $28 / 29$ patients $(90 \%)$ in the Lap-GJ group and $21 / 25$ patients (84\%) in the EUS-GJ group $(\mathrm{P}=0.11)$. Adverse events occurred in $41 \%(\mathrm{n}=12)$ of patients in the Lap-GJ group and $12 \%(n=3)$ in the EUS-GJ group, i.e., significantly lower in the EUS-GJ group $(\mathrm{P}=0.0386)$.

\section{EUS-directed TG ERCP}

ERCP in patients who have undergone Roux-en-Y gastric bypass is technically challenging. The technical success rate of deep enteroscopy-assisted ERCP is 63\%, but this is dependent on the length of the roux limb and operator experience. Ngamruengphong et al [27] describe a multicenter experience using LAMSs (AXIOS and HOT AXIOS Stent) to create an EUS-guided TG fistula to facilitate peroral ERCP in these patients. The technical success rate of EUS-TG was 100\%, from jejunum to the excluded stomach in $54 \%$ of patients and from the gastric pouch to the excluded stomach in the remaining patients. After EUS-TG, ERCP through the LAMS placed through the transgastric fistula was performed successfully in all 13 patients (100\%). Clinical success was achieved in all patients (100\%). Two patients with severe acute cholangitis underwent ERCP during the same session. In 11 patients who underwent ERCP at a subsequent session the LAMS was kept in situ for a median of 11 days prior to the ERCP. Regarding LAMS removal, one of 13 patients still had the stent in place at the time of writing the article. In the remaining 12 patients, the LAMSs were removed at a median of 20 days after EUS-TG.

\section{Postsurgical fluid collections (PSFCs)}

PSFCs are traditionally drained either percutaneously or surgically. Endoscopic drainage offers several advantages compared with either percutaneous or surgical approaches, including avoiding repeat surgery or the need to have a percutaneous drain in place for weeks. Mudireddy et al [28] presented their data about drainage with LAMS ("hot" and "cold" AXIOS in 47 patients with fluid collection after various surgeries). Most collections (26/47, 55\%) were the result of pancreatic duct leaks after pancreatic resections. Other surgical procedures resulting in PSFCs managed with LAMS placement included liver transplantation, liver resection, cholecystectomy, colorectal resection, gynecologic surgery, or bariatric surgery. The site of drainage was transgastric (technical success 32/34), transduodenal (4/5), or transrectal (8/8). The overall technical success rate was $93.6 \%$ and the clinical success rate $89.3 \%$. The rate of intraprocedural adverse events (migrations) was $4.25 \%$ and the postprocedural rate was $6.4 \%$ ( 1 migration, 1 perforation, 1 infection).

\section{Gastrointestinal (GI) benign strictures}

Intuitively, it would seem that LAMS could be used also for selected luminal GI strictures with appropriate anatomy; some recent studies investigated the use of this device in benign luminal GI stricture (BLGS), often refractory to standard endoscopic interventions.

Yang et al [29] presented an observational, retrospective, single-arm, multicenter consecutive case series of patients undergoing LAMS placement for BLGS. Overall, most luminal strictures were anastomotic (25/30;83.3\%), with gastrojejunal anastomotic strictures being the most common type. Median stricture diameter and length were $4.5 \mathrm{~mm}$ and $8 \mathrm{~mm}$, respectively. Technical success was achieved in 29 patients (96.7\%), with an adverse event rate of $13.3 \%$ (one bleeding and one perforation). The stent migration rate was $8.0 \%(2 / 25)$ on follow-up endoscopy. Short-term clinical success was achieved in $90.0 \%$ at a median of 60 days. Most patients (19/23; 82.6\%) experienced sustained symptom improvement or resolution without the need for additional interventions at a median follow up of 100 days after LAMS removal.

\section{Concluding remarks}

EUS connection of luminal structures within the GI tract using LAMSs has gained wide acceptance and has been the subject of many studies with different indications, as these minimally invasive procedures provide great opportunities to avoid invasive surgical procedures. Of the currently available LAMSs, the AXIOS stent is the most commonly described in the literature and has shown very satisfactory rates of technical and clinical success for the procedures described above, in particular for the drainage of PFCs and the bile duct 
system. Because of its cost and the non-negligible adverse events associated with it, further comparative studies with "traditional" techniques are required before LAMSs come into widespread use, but the early results are promising and novel indications will further challenge and expand its applicability.

\section{References}

1. Binmoeller KF, Shah J. A novel lumen-apposing stent for transluminal drainage of nonadherent extraintestinal fluid collections. Endoscopy 2011;43:337-342.

2. Teoh AY, Binmoeller KF, Lau JY. Single-step EUS-guided puncture and delivery of a lumen-apposing stent for gallbladder drainage using a novel cautery-tipped stent delivery system. Gastrointest Endosc 2014;80:1171.

3. Samuelson AL, Shah RJ. Endoscopic management of pancreatic pseudocysts. Gastroenterol Clin North Am 2012;41:47-62.

4. Rinninella E, Kunda R, Dollhopf M, et al. EUS-guided drainage of pancreatic fluid collections using a novel lumen-apposing metal stent on an electrocautery-enhanced delivery system: a large retrospective study (with video). Gastrointest Endosc 2015;82:1039-1046.

5. Shah RJ, Shah JN, Waxman I, et al. Safety and efficacy of endoscopic ultrasound-guided drainage of pancreatic fluid collections with lumen-apposing covered self-expanding metal stents. Clin Gastroenterol Hepatol 2015;13:747-752.

6. Siddiqui AA, Adler DG, Nieto J, et al. EUS-guided drainage of peripancreatic fluid collections and necrosis by using a novel lumen-apposing stent: a large retrospective, multicenter U.S. experience (with videos). Gastrointest Endosc 2016;83:699-707.

7. Walter D, Will U, Sanchez-Yague A, et al. A novel lumen-apposing metal stent for endoscopic ultrasound-guided drainage of pancreatic fluid collections: a prospective cohort study. Endoscopy 2015;47:63-67.

8. Sharaiha RZ, Tyberg A, Khashab MA, et al. Endoscopic therapy with lumen-apposing metal stents is safe and effective for patients with pancreatic walled-off necrosis. Clin Gastroenterol Hepatol 2016;14:1797-1803.

9. Siddiqui AA, Kowalski TE, Loren DE, et al. Fully covered selfexpanding metal stents versus lumen-apposing fully covered selfexpanding metal stent versus plastic stents for endoscopic drainage of pancreatic walled-off necrosis: clinical outcomes and success. Gastrointest Endosc 2017;85:758-765.

10. Yoo J, Yan L, Hasan R, Somalya S, Nieto J, Siddiqui AA. Feasibility, safety, and outcomes of a single-step endoscopic ultrasonographyguided drainage of pancreatic fluid collections without fluoroscopy using a novel electrocautery-enhanced lumen-apposing, selfexpanding metal stent. Endosc Ultrasound 2017;6:131-135.

11. Han D, Inamdar S, Lee CW, Miller LS, Trindade AJ, Sejpal DV. Lumen apposing metal stents (LAMSs) for drainage of pancreatic and gallbladder collections: a meta-analysis. J Clin Gastroenterol 2017 October 9 [Epub ahead of print].

12. Law ST, De La SernaHiguera C, Simón PG, PérezMirandaCastillo M. Comparison of clinical efficacies and safeties of lumen-apposing metal stent and conventional-type metal stent-assisted EUS-guided pancreatic wall-off necrosis drainage: a real-life experience in a tertiary hospital. Surg Endosc 2018;32:2448-2453.

13. Lang GD, Fritz C, Bhat T, et al. EUS-guided drainage of peripancreatic fluid collections with lumen-apposing metal stents and plastic double-pigtail stents: comparison of efficacy and adverse event rates. Gastrointest Endosc 2018;87:150-157.

14. Bang JY, Hasan M, Navaneethan U, Hawes R, Varadarajulu S. Lumen-apposing metal stents (LAMS) for pancreatic fluid collection (PFC) drainage: may not be business as usual. Gut 2017;66:2054-2056.

15. Itoi T, Binmoeller KF. EUS-guided choledochoduodenostomy by using a biflanged lumen-apposing metal stent. Gastrointest Endosc 2014;79:715.

16. Kunda R, Pérez-Miranda M, Will U, et al. EUS-guided choledochoduodenostomy for malignant distal biliary obstruction using a lumen-apposing fully covered metal stent after failed ERCP. Surg Endosc 2016;30:5002-5008.

17. Tsuchiya T, Bun Teoh AY, Itoi T, et al. Long-term outcomes of EUS-guided choledochoduodenostomy using a lumen-apposing metal stent for malignant distal biliary obstruction: a prospective multicenter study. Gastrointest Endosc 2018;87:1138-1146.

18. Anderloni A, Buda A, Carrara S, et al. Single-session doublestent placement in concomitant malignant biliary and duodenal obstruction with a cautery-tipped lumen apposing metal stent. Endoscopy 2016;48:E321-E322.

19. Irani S, Baron TH, Grimm IS, Khashab MA. EUS-guided gallbladder drainage with a lumen-apposing metal stent (with video). Gastrointest Endosc 2015;82:1110-1115.

20. Walter D, Teoh AY, Itoi T, et al. EUS-guided gall bladder drainage with a lumen-apposing metal stent: a prospective long-term evaluation. Gut 2016;65:6-8.

21. Dollhopf M, Larghi A, Will U, et al. EUS-guided gallbladder drainage in patients with acute cholecystitis and high surgical risk using an electrocautery-enhanced lumen-apposing metal stent device. Gastrointest Endosc 2017;86:636-643.

22. Teoh AYB, Serna C, Penas I, et al. Endoscopic ultrasoundguided gallbladder drainage reduces adverse events compared with percutaneous cholecystostomy in patients who are unfit for cholecystectomy. Endoscopy 2017;49:130-138.

23. Irani S, Ngamruengphong S, Teoh A, et al. Similar efficacies of endoscopic ultrasound gallbladder drainage with a lumenapposing metal stent versus percutaneous transhepatic gallbladder drainage for acute cholecystitis. Clin Gastroenterol Hepatol 2017; 15:738-745.

24. Imai $\mathrm{H}$, Kitano $\mathrm{M}$, Omoto $\mathrm{S}$, et al. EUS-guided gallbladder drainage for rescue treatment of malignant distal biliary obstruction after unsuccessful ERCP. Gastrointest Endosc 2016;84:147-151.

25. Tyberg A, Perez-Miranda M, Sanchez-Ocaña R, et al. Endoscopic ultrasound-guided gastrojejunostomy with a lumen-apposing metal stent: a multicenter, international experience. Endosc Int Open 2016;4:E276-E281.

26. Khashab MA, Bukhari $\mathrm{M}$, Baron $\mathrm{TH}$, et al. International multicenter comparative trial of endoscopic ultrasonographyguided gastroenterostomy versus surgical gastrojejunostomy for the treatment of malignant gastric outlet obstruction. Endosc Int Open 2017;5:E275-E281.

27. Ngamruengphong S, Nieto J, Kunda R, et al. Endoscopic ultrasound-guided creation of a transgastric fistula for the management of hepatobiliary disease in patients with Roux-en-Y gastric bypass. Endoscopy 2017;49:549-552.

28. Mudireddy PR, Sethi A, Siddiqui AA, et al. EUS-guided drainage of postsurgical fluid collections using lumen-apposing metal stents: a multicenter study. Gastrointest Endosc 2018;87:1256-1262.

29. Yang D, Nieto JM, Siddiqui A, et al. Lumen-apposing covered selfexpandable metal stents for short benign gastrointestinal strictures: a multicenter study. Endoscopy 2017;49:327-333. 